Research Article

\title{
Bioinformatics Analysis Reveals MCM3 as an Important Prognostic Marker in Cervical Cancer
}

\author{
Hui Ma $\mathbb{D},{ }^{1}$ Zhen Liu, ${ }^{2}$ Honglin Li, ${ }^{1}$ Xuewang Guo, ${ }^{1}$ Sujie Guo, ${ }^{1}$ Pengpeng Qu $\mathbb{D},{ }^{3}$ \\ and Yuquan Wang iD 1 \\ ${ }^{1}$ Department of Gynecology, The Secondary Hospital of Tianjin Medical University, No. 23 Pingjiang Road, Hexi District, \\ Tianjin 300211, China \\ ${ }^{2}$ Department of Gynecology, Chifeng Municipal Hospital, Chifeng Clinical Medical School of Inner Mongolia Medical University, \\ Chifeng 024099, China \\ ${ }^{3}$ Department of Gynecology Oncology, Tianjin Central Hospital of Gynecology \& Obstetrics, 156 Sanmalu, Nankai, \\ Tianjin 300100, China
}

Correspondence should be addressed to Pengpeng Qu; qu.pengpeng@hotmail.com and Yuquan Wang; spring93@163.com

Received 12 August 2021; Revised 14 September 2021; Accepted 18 September 2021; Published 11 October 2021

Academic Editor: Jialiang Yang

Copyright (C) 2021 Hui Ma et al. This is an open access article distributed under the Creative Commons Attribution License, which permits unrestricted use, distribution, and reproduction in any medium, provided the original work is properly cited.

\begin{abstract}
The minichromosome maintenance complex 3 (MCM3) is essential for the regulation of DNA replication and cell cycle progression. However, the expression and prognostic values of MCM3 in cervical cancer (CC) have not been well-studied. Herein, we investigated the expression patterns and survival data of MCM3 in cervical cancer patients from the ONCOMINE, GEPIA, Human Protein Atlas, UALCAN, Kaplan-Meier Plotter, and LinkedOmics databases. The expression level of MCM3 is negatively correlated with advanced tumor stage and metastatic status. Specifically, MCM3 is significantly differentially expressed between patients in stage 1 and stage 3 cervical cancer with $p$ value 0.0138 . Similarly, the $p$ values between stage 1 and stage 4 cervical cancer, between stage 2 and stage 3 , and between stage 2 and stage 4 are $0.00089,0.0244$, and 0.00197, respectively. Not only that, cervical cancer patients with high mRNA expression of MCM3 may indicate longer overall survival but indicate shorter relapse-free survival. PRIM2 and MCM6 are positively correlated genes of MCM3. Bioinformatics analysis revealed that MCM3 might be considered a biological indicator for prognostic evaluation of cervical cancer. However, it is currently limited to bioinformatics analysis, and more clinical tissue specimens and cell experiments are needed to further explore the role of MCM3 in the occurrence and progression of cervical cancer.
\end{abstract}

\section{Introduction}

Cervical cancer is a common gynecological tumor. The incidence rate and mortality rate rank fourth in the female malignant tumor worldwide, only behind breast, colorectal, and lung cancer [1]. However, in developing countries, the incidence and mortality of cervical cancer rank second only to breast cancer in female malignant tumors [2]. Cervical cancer is one of the cancers that can be prevented through screening. With the widespread popularity of screening, the incidence of cervical cancer is declining year by year. However, as early cervical cancer has no symptoms, many patients are already in the middle and advanced stages of disease when they were diagnosed [3]. The main treatments for cervical cancer include surgery, radiotherapy, and adjuvant platinum-based chemotherapy. Early cervical cancer is mainly treated by surgery, and the 5-year survival rate can reach $88-95 \%$. However, there are limited treatment methods for patients in the middle and advanced stages, and the therapeutic effect of radiotherapy and chemotherapy is not satisfactory [4-6]. Therefore, it is urgently needed to discover novel molecular biomarkers, therapeutic targets, or prognostic evaluation index for cervical cancer. The extensive application of bioinformatics databases has facilitated the discovery of new biomarkers for cancer management [7-10].

The minichromosome maintenance complex (MCM) family proteins (MCM2-8 and MCM10) play an essential 


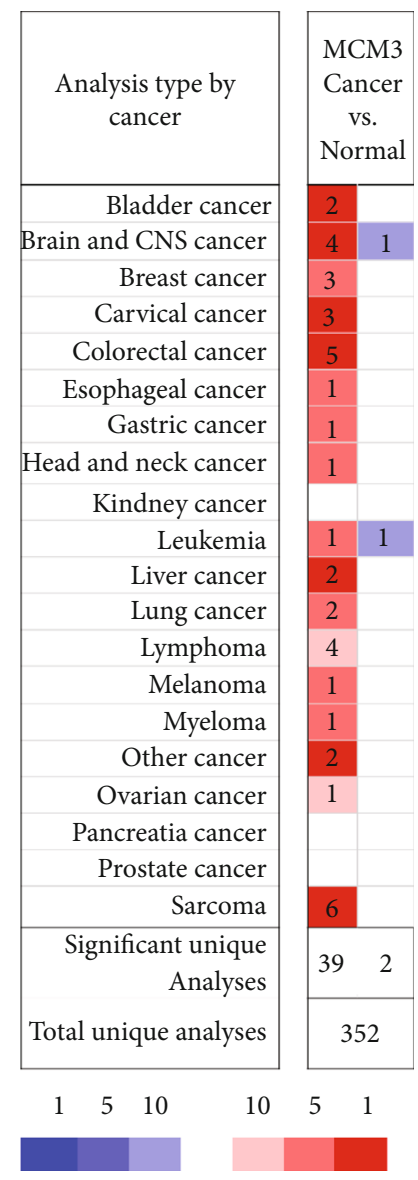

(\%)

(a)

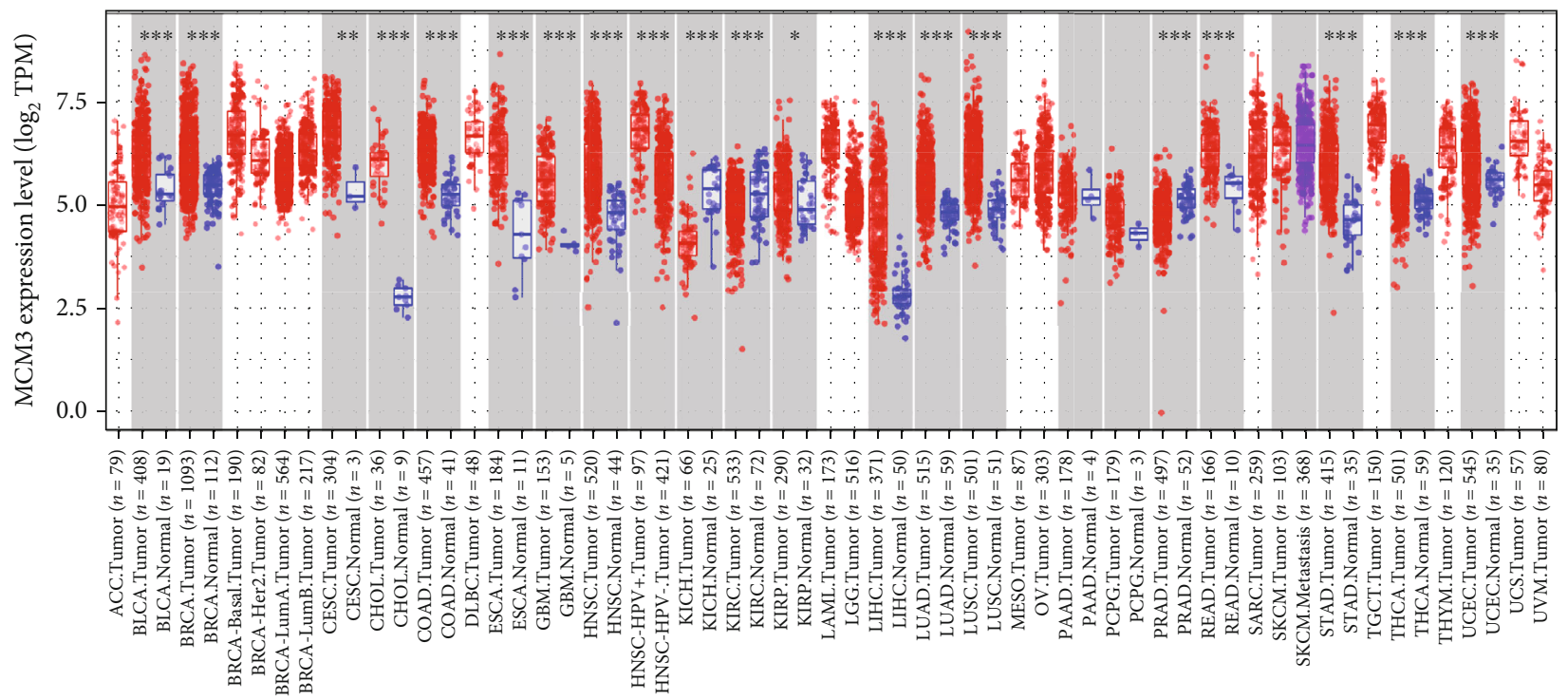

(b)

FIGURE 1: MCM3 expression levels across cancers. (a) The transcription levels of MCM3 across cancers. The figure is generated from ONCOMINE with exact thresholds ( $p$ value: IE-4; fold change: 2; gene rank: top 10\%). (b) MCM3 expression levels across cancers from TCGA data in TIMER 2. ${ }^{*} p<0.05,{ }^{* *} p<0.01$, and ${ }^{* * *} p<0.001$. 


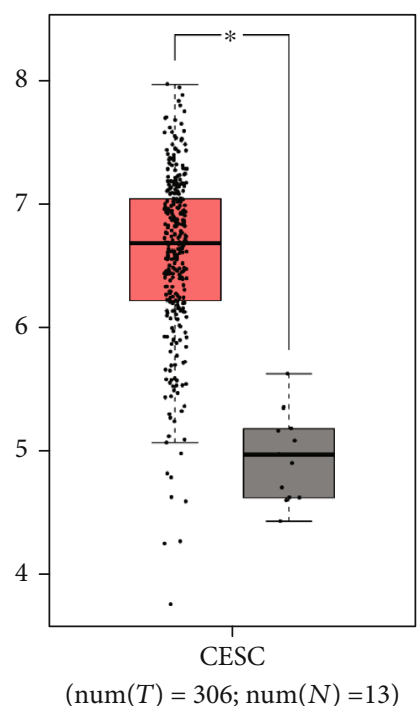

(a)

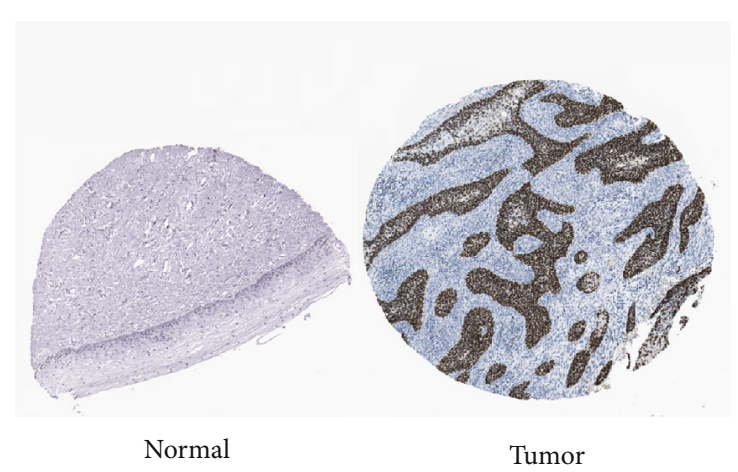

(b)

Figure 2: The expression of MCM3 in cervical cancer: (a) GEPIA box plot; (b) IHC of MCM3 in normal (N) and tumor (T) tissues from patients with cervic al cancer in the Human Protein Atlas.

TABle 1: Datasets of MCM3 expression in cervical cancers (ONCOMINE database).

\begin{tabular}{lccccc}
\hline Cancer site & Types of cancer vs. normal & Fold change & $t$-test & $p$ value & Dataset \\
\hline MCM3 & Cervical cancer vs. normal & 4.180 & 11.188 & $2.84 E-13$ & Pyeon \\
& Cervical squamous cell carcinoma vs. normal & 2.613 & 7.947 & $1.05 E-10$ & Scotto \\
& Cervical squamous cell carcinoma vs. normal & 2.589 & 12.088 & $1.48 E-6$ & Biewenga \\
\hline
\end{tabular}

role in the initiation of the DNA replication process and cell division [11, 12]. MCM2-7 complex acts as a helicase during the initiation of DNA replication while MCM8 performs a similar uncoiling effect during the elongation of DNA replication. In addition, MCM10 participates in the regulation of DNA replication elongation $[13,14]$. MCM3 is one of the MCM families. MCM3 acetylation could initiate DNA replication and cell cycle progression [15]. MCM3 expression is upregulated in a variety of malignant tumor cells [16]. Moreover, the expression of MCM3 is deregulated in several human malignancies, including lung cancer [17], colorectal cancer [18], liver cancer [19], breast cancer [20], ovarian cancer [21], and oral squamous cell carcinoma [22]. Zhou et al. found that colorectal cancer patients with high expression of MCM3 have a poor prognosis [18]. A study of liver cancer showed that high expression of MCM3 was associated with tumor invasion and poor prognosis of liver cancer patients [19]. Løkkegaard et al. found that ER+ breast cancer cells can develop resistance to tamoxifen and letrozole by upregulating MCM3 [20].

Despite the intensive study of MCM3 in many cancers, there is limited research regarding MCM3 in cervical cancer. By studying the role of MCM3 in cervical cancer, it might provide a novel biomarker for drug development and repositioning on this cancer.

\section{Methods}

2.1. ONCOMINE Analysis. ONCOMINE (https://www .oncomine. org/resource/login.html) is an online bioinfor- matics database [23]. In this study, we used ONCOMINE to analyze the transcription levels of MCM3 mRNA and compare the expression of MCM3 in cervical cancer and normal cervical tissues, Shan parameters: $p<\mathrm{IE}-4$, fold change $>2$, and gene rank: $10 \%$.

2.2. GEPIA Dataset. GEPIA (http://gepia.cancer-pku.cn/) is an interactive web server for analyzing the RNA sequencing expression data from TCGA and GTEx projects [24, 25]. We used GEPIA to compare the expression of MCM3 mRNA in cervical tumor/normal tissues. The differences in transcription expression was determined by Student's $t$-test, and $p$ values $<0.05$ were considered statistically significant.

2.3. TIMER 2. TIMER (https://cistrome.shinyapps.io/timer/) is a comprehensive resource for the systematic analysis of immune infiltrates [26]. We used TIMER to analyze the correlation between the expression of MCM3 in cervical cancer and the abundance of the six immune infiltrates, including $\mathrm{B}$ cells, CD4+ T cells, CD8+ T cells, neutrophils, macrophages, and dendritic cells in cervical cancer. The scatterplots show purity-corrected partial Spearman's rho.

2.4. Human Protein Atlas. The Human Protein Atlas (https://www.proteinatlas.org/) is aimed at mapping all the human protein in cells, tissues, and organs, including antibody-based imaging [27]. We use this database to retrieve immunohistochemical staining pictures of MCM3 in cervical cancer and normal cervix. 


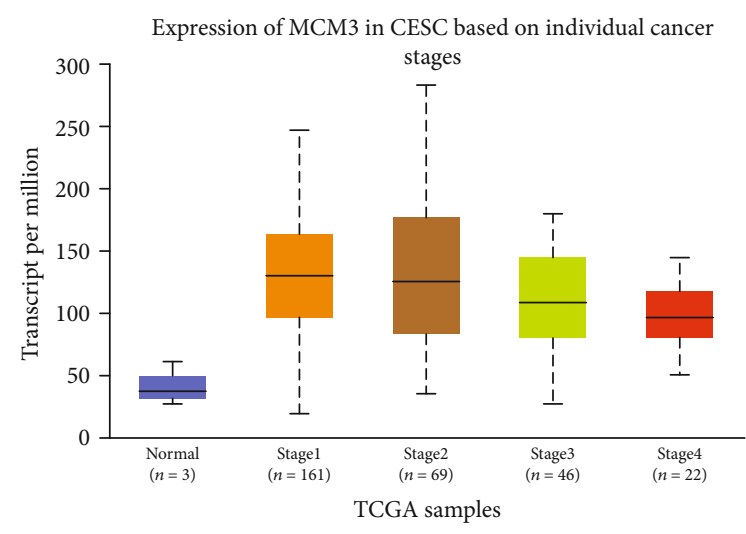

(a)

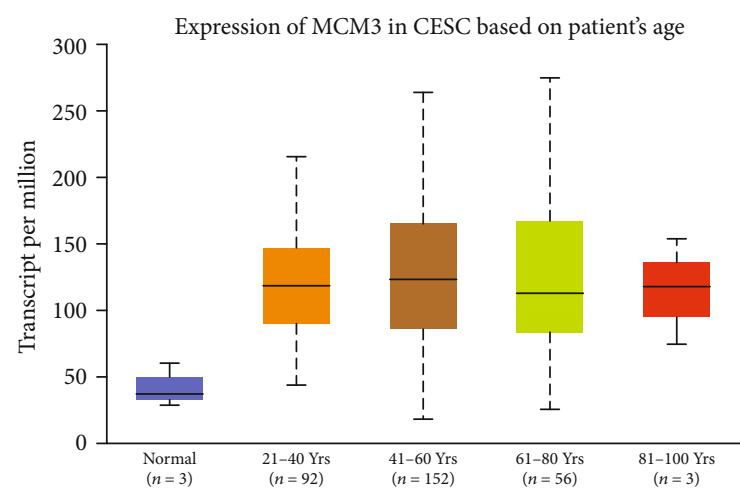

(c)

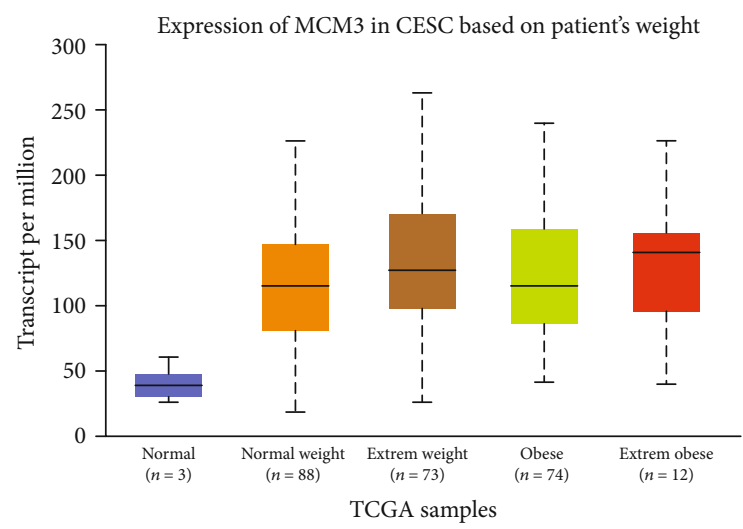

(e)

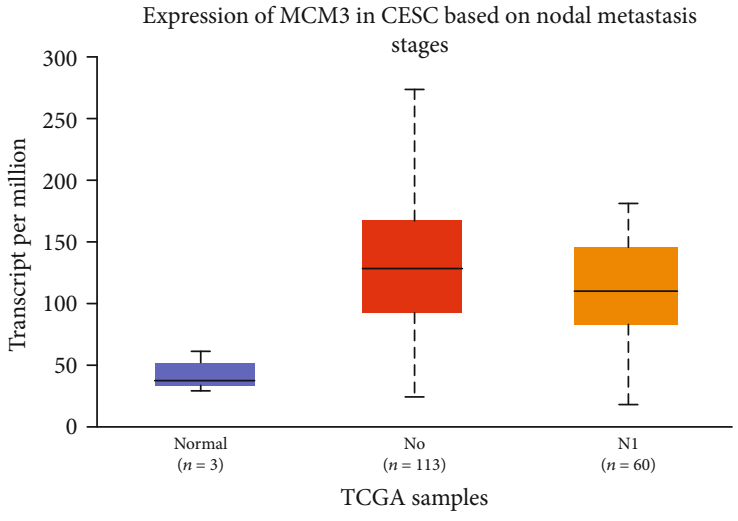

(b)

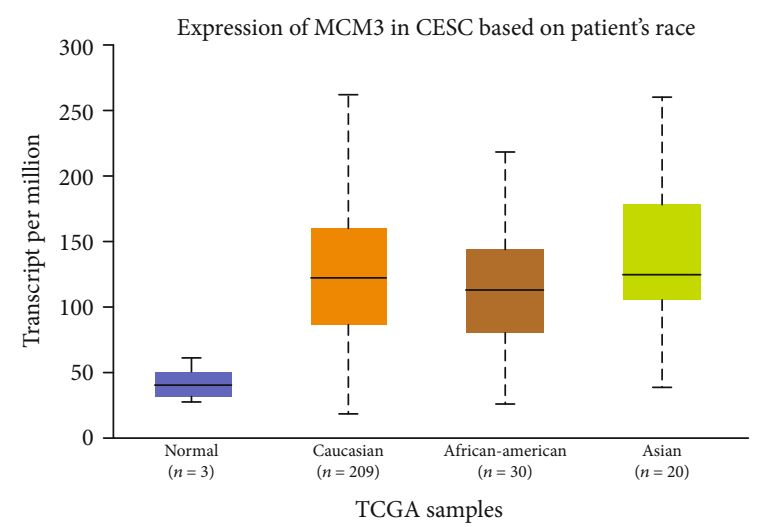

(d)

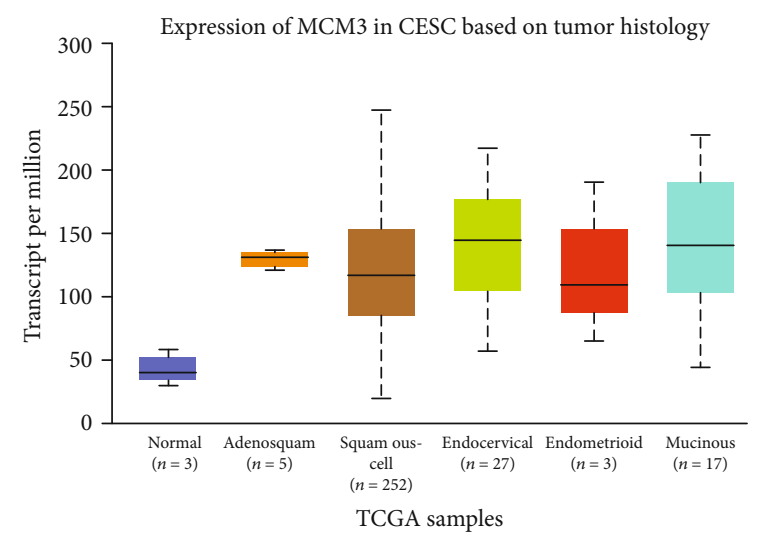

(f)

Figure 3: Continued. 

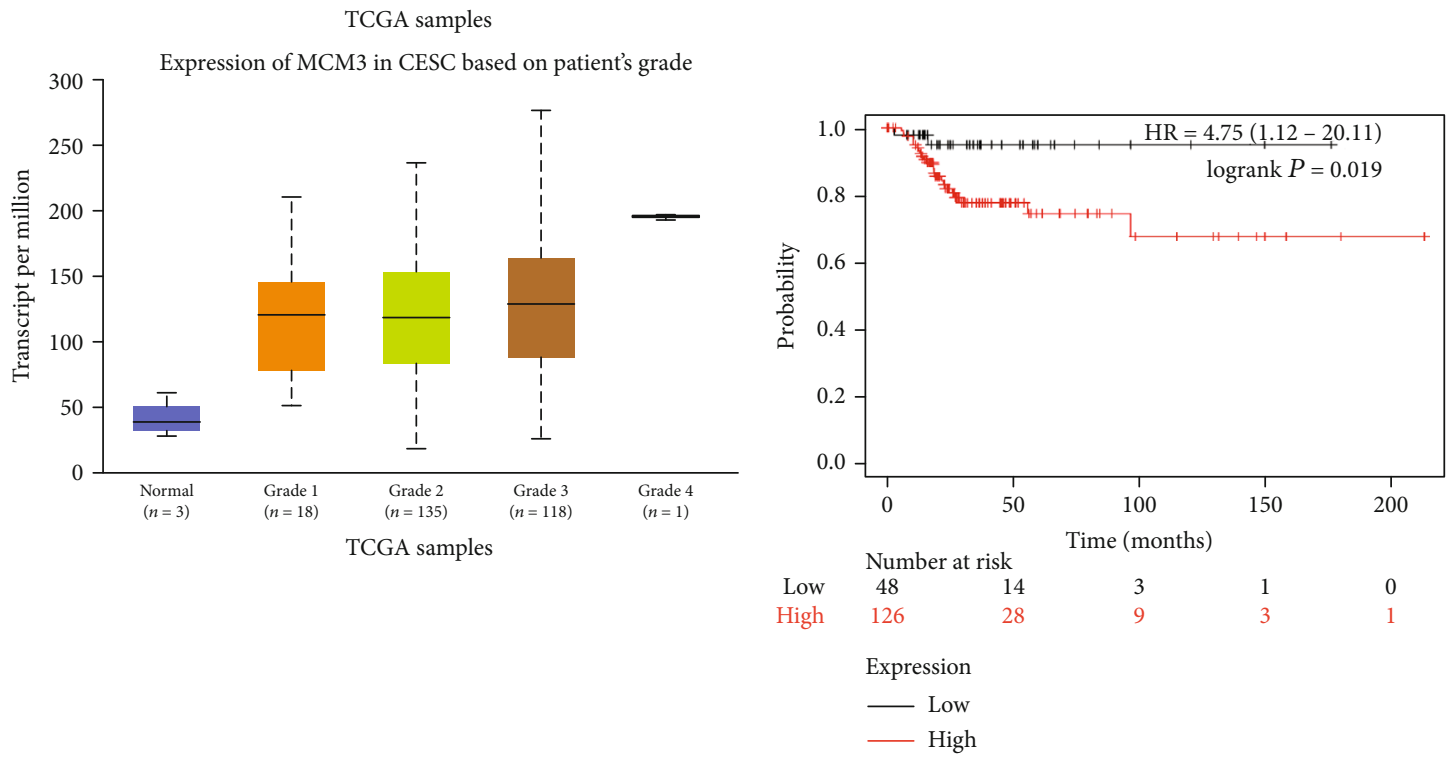

(g)

(h)

FIGURE 3: Box plot revealing the relationship between MCM3 expression and different clinical indicators using the UALCAN cancer database shown for stage (a), nodal metastasis (b), age (c), race (d), weight (e), tumor histology (f), and tumor grade (g); analysis is shown for OS (h) and RFS (i) by the Kaplan-Meier Plotter.

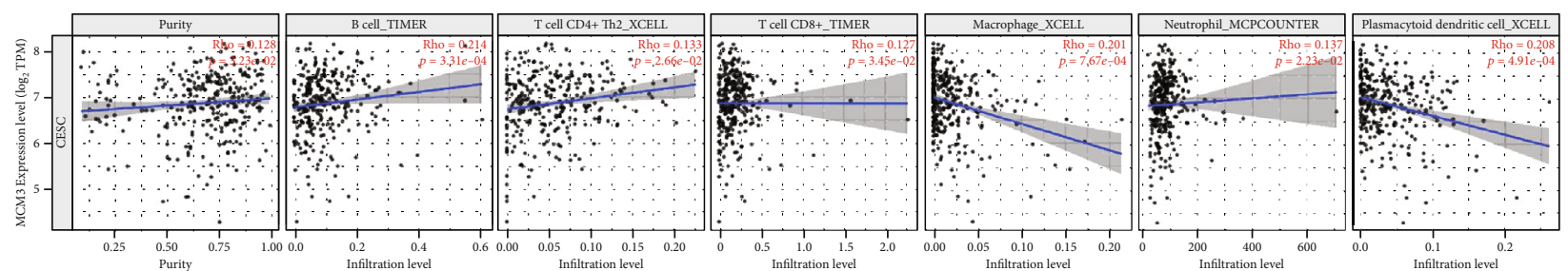

FIgure 4: The correlation between MCM3 expression and immune infiltration level in CC. Correlation of MCM3 expression with tumor purity and infiltrating levels of B cell, CD8+ T cell, CD4+ T cell, macrophage, neutrophil, and dendritic cell in CC. $p<0.05$ is considered statistically significant. CC: cervical cancer.

2.5. Kaplan-Meier Plotter. Kaplan-Meier Plotter (https:// kmplot.com/analysis/) is a large sample-based dataset that is able to evaluate the survival of patients with different types of cancers [28]. We used the Kaplan-Meier Plotter to analyze the prognostic value of MCM3 expression in cervical cancer. $p<0.05$ was considered statistically significant. The hazard ratios (HRs) with specific 95\% confidence intervals (CIs) and $p$ values were listed.

2.6. UALCAN Cancer Database. UALCAN (http://ualcan .path.uab.edu/) uses data from the Clinical Proteomic Tumor Analysis Consortium (CPTAC) Confirmatory/Discovery dataset to provide protein expression analysis option [29]. We evaluated the expression of MCM3 in cervical cancer by TCGA analysis.

2.7. LinkedOmics Dataset. LinkedOmics (http://www .linkedomics.orglogin.php) is a new and unique datasets for disseminating data from large-scale cancer omics projects [30]. We used this database to find genes closely related to MCM3.

\section{Results}

3.1. MCM3 Expression Level across Cancers. We used the ONCOMINE database to compare the expression levels of MCM3 mRNA across cancers and its mRNA expression with that in corresponding normal tissues. Figure 1(a) presents the expression of the MCM3 across cancers. The results showed that MCM3 expression was higher in several cancer groups than in normal tissues, including the bladder, brain, breast, cervical, colorectal, liver, head and neck, and lung, as well as lymphoma. However, the mRNA expression of MCM3 was significantly downregulated in leukemia and brain cancers. Moreover, we further used TIMER to evaluate the expression of MCM3 in TCGA. Figure 1(b) shows the details of MCM3 expression across cancers.

3.2. Overexpression of MCM3 in Cervical Cancer. ONCOMINE and GEPIA datasets were used to compare the expression level of MCM3 in cervical cancer with those in corresponding normal tissues. We further used the Human Protein Atlas database to retrieve immunohistochemical 


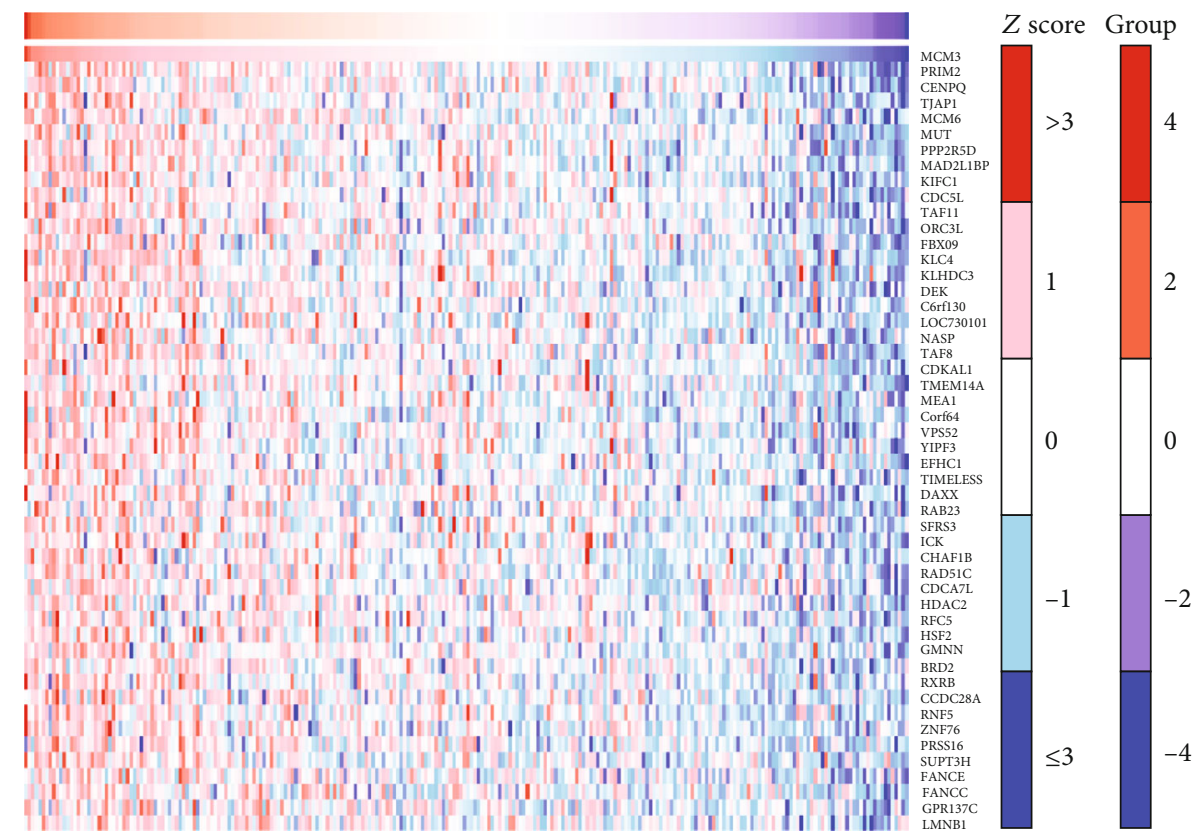

(a)

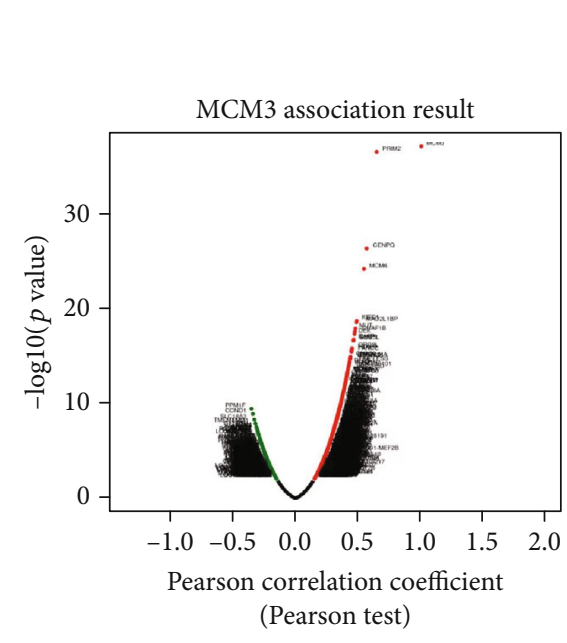

(b)

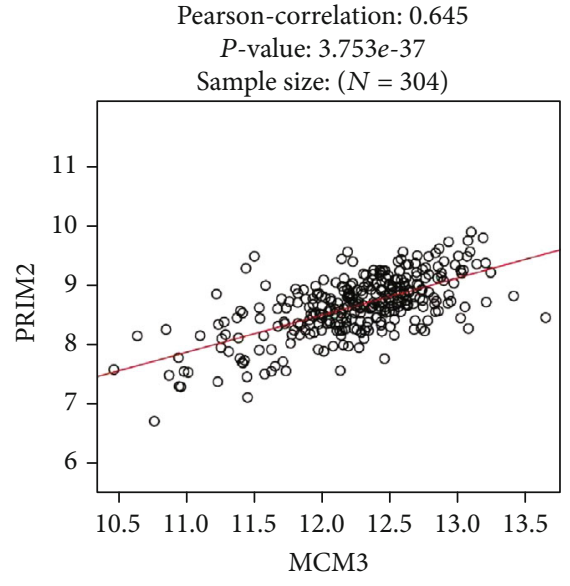

(c)

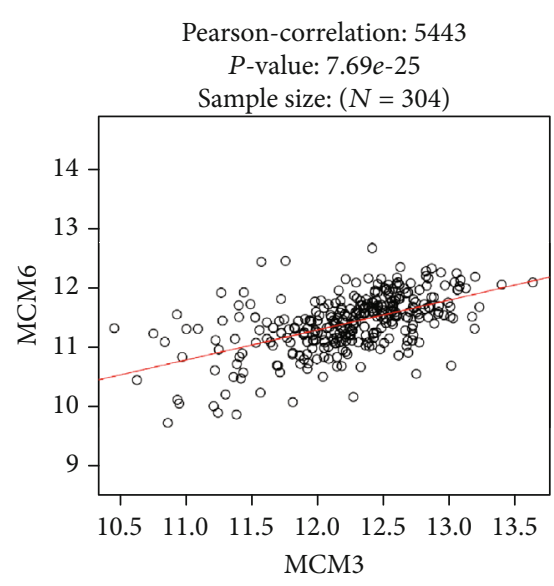

(d)

FIGURE 5: Coexpression analysis of MCM3: $(a, b)$ coexpression profile of MCM3 identified using the LinkedOmics dataset; (c) the correlation between MCM3 and PRIM2 expression in cervical cancer by the LinkedOmics dataset; (d) the correlation between MCM3 and MCM6 expression in cervical cancer by the LinkedOmics dataset.

staining pictures of MCM3 in cervical tissues. Figure 2(a) presents the expression of the MCM3 in cervical cancer compared with normal tissues. MCM3 were significantly upregulated in cervical cancer tissues. The details are shown in Table $1[25,31,32]$. In addition, we compared the MCM3 protein expression level in cervical cancer and normal cervical tissues through the Human Protein Atlas datasets (Figure 2(b)).

3.3. Relationship between the Expression Levels of MCM3 $m R N A$ and the Clinicopathological Parameters of Patients with Cervical Cancer. We used the GEPIA dataset and UALCAN cancer database to analyzed clinicopathological parameters for cervical cancer. MCM3 expression was sig- nificantly related to tumor stage (Figure $3(\mathrm{a}), p<0.001$ ) and lymph node metastasis (Figure $3(\mathrm{~b}), p<0.001$ ), whereas age (Figure $3(\mathrm{c}), p>0.05)$, race (Figure $3(\mathrm{~d}), p>0.05$ ), weight (Figure 2(e), $p>0.05$ ), tumor histology (Figure 3(f), $p>0.05$ ), and tumor grade (Figure $3(\mathrm{~g}), p>0.05$ ) were not significantly associated. We further analyzed the prognostic value of MCM3 in cervical cancers in the Kaplan-Meier Plotter. The detailed results are shown in Figure 3(h). The Kaplan-Meier curve and log rank test analyses revealed that the increased MCM3 mRNA levels were significantly associated with better overall survival (OS) $(p<0.05)$ of all of the patients with cervical cancer. On the contrary, increased MCM3 mRNA levels were significantly associated with shorter relapse-free survival (RFS) $(p<0.05)$. 
3.4. Correlations between MCM3 Expression and Immune Infiltration in CC. Immune infiltration in the tumor microenvironment can affect the survival of patients. Next, we analyzed the correlation between the expression of MCM3 in cervical cancer and immune infiltration. Cervical cancer patients had good survival with high MCM3 expression. We used the TIMER database to explore the correlation between MCM3 and immune cell infiltration in cervical cancer. The results showed that MCM3 was significantly related to the purity of cervical cancer $(R=0.128, p=3.23 e-02)$. The expression of MCM3 was significantly positively correlated with the infiltration level of CD4+ T cells $(R=0.133$, $p=2.66 e-02)$, neutrophils $(R=0.137, p=2.23 e-02), \mathrm{B}$ cells $(R=0.214, p=3.31 e-04)$, and CD8+ T cells $(R=$ $0.127, p=3.45 e-02)$, while the expression of MCM3 was significantly negatively correlated with the infiltration level of macrophages $(R=-0.201, p=7.67 e-04)$ and dendritic cells $(R=-0.208, p=4.91 e-04)$ in cervical cancers (Figure 4$)$.

3.5. Correlation Analysis. In order to further study the potential mechanism of MCM3 in cervical cancer, we excavated the gene data coexpressed with MCM3 by the GEPIA database and LinkedOmics database. PRIM2 is a correlated gene (Figures 5(a) and 5(b), Table 2). Further analysis using the GEPIA database and LinkedOmics database revealed the correlation between MCM3 and PRIM2 or MCM6. Pearson correlation analysis showed that the expression of MCM3 and PRIM2 or MCM6 in cervical cancer was obviously positive $(R=0.6387$ and $R=0.5443$, respectively, Figures 5(c) and $5(\mathrm{~d}))$.

PRIM2 and MCM6 were significantly upregulated in cervical cancer tissues by the GEPIA database (Figures 6(a) and $6(\mathrm{~d})$ ). We further used the Kaplan-Meier plotter database to analyze the survival of cervical cancer patients, and the analysis results suggest that the upregulation of PRIM2 and MCM6 is related to the longer OS of cervical cancer patients (Figures 6(b) and 6(e)), while only MCM6 is related to shorter RFS, which is similar to MCM3.

\section{Discussion}

Previous studies have shown overexpression of MCM3 in many cancers [15-18, 20, 21]. Zhou et al. [18] found that elevated MCM3 expression was associated with poor prognosis of CRC patients. They further confirmed that MCM3 was overexpressed in CRC cell lines through vitro experiments. In addition, knockdown of MCM3 in CRC cells could significantly inhibit CRC cell proliferation, migration, invasion, and transition of the G1 to S phase. Decreasing the expression of MCM3 in endocrine-resistant cells restored drug sensitivity, and detecting the expression of MCM3 may predict the response of patients to endocrine treatment [20]. Another study in ovarian cancer showed that increased expression of MCM-3 and Ki-67 was associated with increased histological malignancy [21]. Compared with controls, MCM3 were highly expressed in CSCC, and MCM3 expression was correlated with CSCC cell differentiation, while, in terms of prognostic value assessment, there is no independent prognostic correlation between MCM3 and
TABLE 2: Coexpression profile of MCM3 identified using the GEPIA database.

\begin{tabular}{lcc}
\hline Gene symbol & \multicolumn{1}{c}{ Gene ID } & PCC \\
\hline PRIM2 & ENSG00000146143.17 & 0.75 \\
MCM6 & ENSG00000076003.4 & 0.69 \\
MUT & ENSG00000146085.7 & 0.68 \\
CENPQ & ENSG00000031691.6 & 0.66 \\
TIMELESS & ENSG00000111602.11 & 0.65 \\
DEK & ENSG00000124795.14 & 0.65 \\
SRSF3 & ENSG00000112081.16 & 0.64 \\
TJAP1 & ENSG00000137221.14 & 0.63 \\
TXNDC16 & ENSG00000087301.8 & 0.63 \\
RBMX & ENSG00000147274.14 & 0.63 \\
\hline
\end{tabular}

clinicopathological parameters [33]. Although the role of MCM3 in the tumorigenesis and prognosis of several cancers has been partially confirmed, further bioinformatics analysis of cervical cancer has yet to be performed. Importantly, the current study focuses on the analysis of exploring the expression and prognostic values of MCM3 in cervical cancer. We hope that the current findings will contribute to provide new ideas for the clinical diagnosis, prognosis assessment, and targeted therapy of cervical cancer. In our study, ONCOMINE datasets and the GEPIA database revealed that the expression of MCM3 was significantly higher in cervical cancer than in normal tissues. MCM3 expression was significantly related to tumor stage $(p<0.001)$ and lymph node metastasis $(p<0.001)$. A high MCM3 expression was significantly associated with better $\mathrm{OS}$ in patients with cervical cancer.

The tumor microenvironment (TME) could affect the progression and recurrence of tumors and has received increasing attention. Immune cells are considered an important determinant of clinical outcome and immunotherapy response [34]. Our study shows that the expression of MCM3 may be significantly related to the infiltration of six immune cell types, indicating that MCM3 may also reflect the immune status in addition to the prognosis of the disease. This research may provide detailed immune information to help design new immunotherapies.

We further searched genes closely related to MCM3 through the GEPIA database, UALCAN cancer database, and LinkedOmics. We selected two proteins PRIM2 and MCM6, which have strong correlation with MCM3 and have different expressions in cervical cancer compared with normal cervical tissues. Through analysis, we found that the expression of PRIM2 and MCM6 was positively correlated with MCM3 expression. We further evaluate their prognostic value in cervical cancer.

PRIM2 is a large subunit of DNA primase, located at 6 11.1-p12 of the human chromosome $[35,36]$. The upregulated expression of PRIM2 in cervical cancer enhanced DNA synthesis, accelerated the progression of cell cycle from the $\mathrm{G} 1$ to $\mathrm{S}$ phase, and promoted the proliferation of cervical cancer cells and the growth of cervical cancer [37]. MCM6 is a number of the minichromosome maintenance 

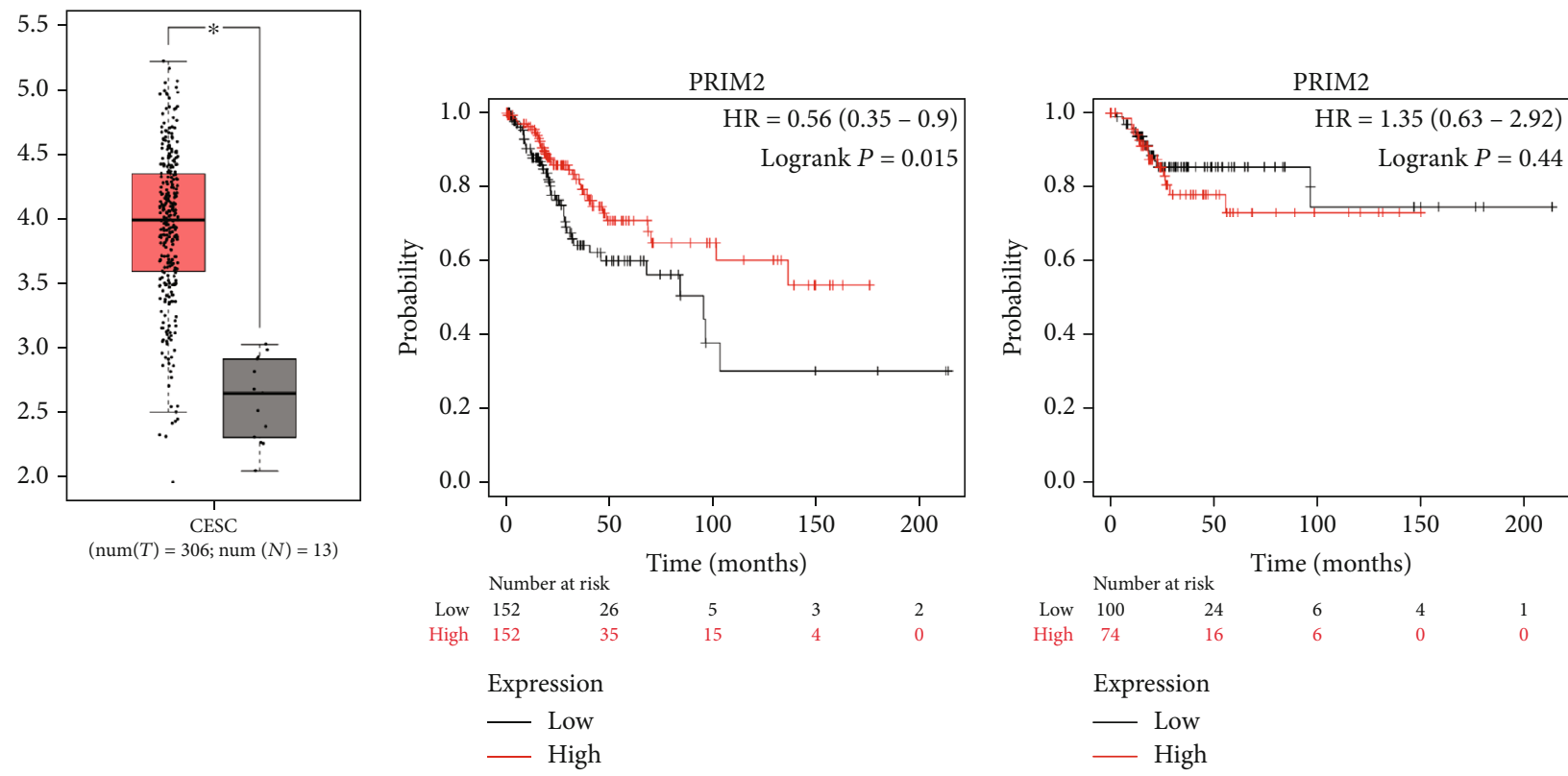

(a)

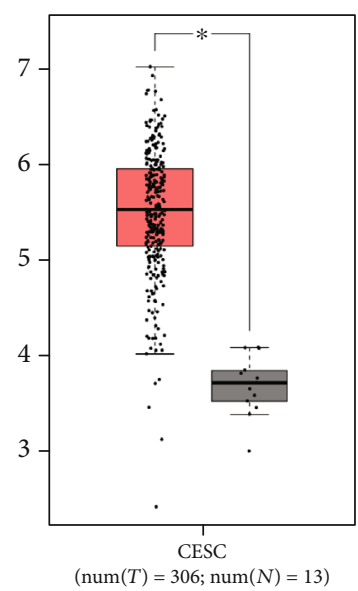

(d)

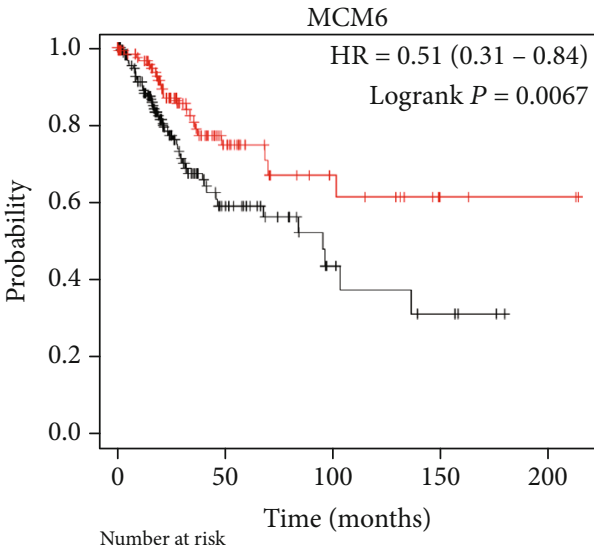

$$
\begin{array}{rlr} 
& \multicolumn{2}{l}{\text { Number at risk }} \\
\text { Low } & 173 \\
\text { High } & 131 \\
& \text { Expression } \\
& \\
& \\
&
\end{array}
$$

(b)

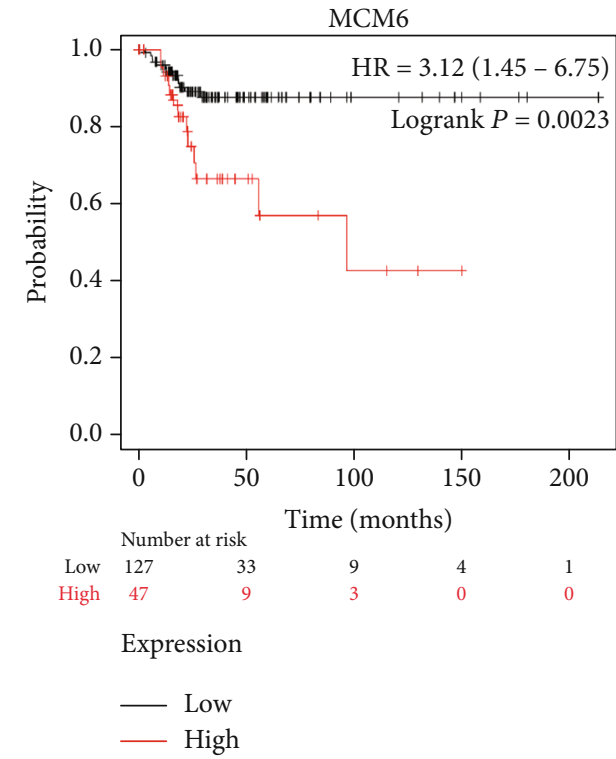

(e) (c)

(f)

FIGURE 6: PRIM2 and MCM6 in cervical cancer: (a) the expression of PRIM2 showed by the GEPIA database; (b) the OS status for the expression of PRIM2 from the Kaplan-Meier Plotter; (c) the RFS status for the expression of PRIM2 from the Kaplan-Meier Plotter; (d) the expression of MCM6 showed by the GEPIA database; (e) the OS status for the expression of MCM6 from the Kaplan-Meier Plotter; (f) the RFS status for the expression of MCM6 from the Kaplan-Meier Plotter.

complex (MCM) family proteins $[11,13]$. In a study of lung cancer [38], the high expression of MCM6 suggests a short overall survival. However, contrary to the conclusions of this study, we found that patients with high expression of MCM6 have a better overall survival but have a poor disease progression-free survival.

In summary, this analysis shows that MCM3 is more highly expressed in cervical cancer than normal cervical tissues. The results indicate that MCM3, PRIM2, and MCM6 could be used for early detection of cervical cancer and may be used as indicators of prognosis. Moreover, MCM3 may become the target of immunotherapy for cervical cancer in the future.

However, our current research has some limitations. All the data analyzed in this study are from bioinformatics databases. We will further explore the potential mechanism of MCM3 in cervical cancer by clinical tissues and cervical cancer cell lines in in vivo and in vitro experiments. 


\section{Data Availability}

The data used in this study was downloaded from TCGA website under the selection of cervical cancer (https:// portal.gdc.cancer.gov/).

\section{Conflicts of Interest}

The authors declare that there is no conflict of interest regarding the publication of this article.

\section{Authors' Contributions}

Hui Ma, Zhen Liu, Yuquan Wang, and Pengpeng Qu conceived and designed the project. Honglin $\mathrm{Li}$ and Xuewang Guo researched literature. Hui Ma, Honglin Li, and Sujie Guo analyzed experimental results. Hui Ma wrote the manuscript. All authors read and gave their approval for the final version of the manuscript. Hui Ma, Zhen Liu, Honglin Li, and Xuewang Guo contributed equally to this work.

\section{Acknowledgments}

This work was supported by the Second Hospital of Tianjin Medical University Youth Fund Project (grant No. 2019ydey19).

\section{References}

[1] W. Yang, Y. Liu, R. Dong et al., "Accurate detection of HPV integration sites in cervical cancer samples using the nanopore MinION sequencer without error correction," Frontiers in Genetics, vol. 11, 2020.

[2] F. Bray, J. Ferlay, I. Soerjomataram, R. L. Siegel, L. A. Torre, and A. Jemal, "Global cancer statistics 2018: GLOBOCAN estimates of incidence and mortality worldwide for 36 cancers in 185 countries," CA: a Cancer Journal for Clinicians, vol. 68, no. 6, pp. 394-424.

[3] R. L. Siegel, K. D. Miller, H. E. Fuchs, and A. Jemal, "Cancer statistics, 2021," CA: a Cancer Journal for Clinicians, vol. 71, no. 1, pp. 7-33, 2021.

[4] P. Basu, K. Taghavi, S.-Y. Hu, S. Mogri, and S. Joshi, "Management of cervical premalignant lesions," Current Problems in Cancer, vol. 42, no. 2, pp. 129-136, 2018.

[5] S. Ma, J. Wang, Y. Han et al., "Platinum single-agent vs. platinum-based doublet agent concurrent chemoradiotherapy for locally advanced cervical cancer: a meta-analysis of randomized controlled trials," Gynecologic Oncology, vol. 154, no. 1, pp. 246-252, 2019.

[6] P. Y. Hou, C. H. Hsieh, M. C. Wei, S. M. Hsiao, and P. W. Shueng, "Differences in treatment outcomes and prognosis between elderly and younger patients receiving definitive radiotherapy for cervical cancer," International Journal of Environmental Research and Public Health, vol. 17, no. 12, p. 4510, 2020.

[7] J. Yang, T. G. T. E. Consortium, T. Huang et al., "Synchronized age-related gene expression changes across multiple tissues in human and the link to complex diseases," Scientific Reports, vol. 5, no. 1, 2015.
[8] J. Yang, S. Peng, B. Zhang et al., "Human geroprotector discovery by targeting the converging subnetworks of aging and agerelated diseases," Geroscience, vol. 42, no. 1, pp. 353-372, 2020.

[9] C. Liu, D. Wei, J. Xiang et al., "An improved anticancer drugresponse prediction based on an ensemble method integrating matrix completion and ridge regression," Mol Ther Nucleic Acids, vol. 21, pp. 676-686, 2020.

[10] X. Xu, H. Long, B. Xi et al., "Molecular network-based drug prediction in thyroid cancer," International Journal of Molecular Sciences, vol. 20, no. 2, p. 263, 2019.

[11] E. M. Johnson, K. Yayoi, and D. C. Daniel, “A new member of the MCM protein family encoded by the human MCM8 gene, located contrapodal to GCD10 at chromosome band 20p12.313," Nucleic Acids Research, vol. 31, no. 11, pp. 2915-2925, 2003.

[12] M. L. Bochman and A. Schwacha, "The Mcm complex: unwinding the mechanism of a replicative helicase," Microbiology and Molecular Biology Reviews: $M M B R$, vol. 73, no. 4, pp. 652-683, 2009.

[13] D. Maiorano, O. Cuvier, E. Danis, and M. Méchali, "MCM8 is an MCM2-7-related protein that functions as a DNA helicase during replication elongation and not initiation," Cell, vol. 120, no. 3, pp. 315-328, 2005.

[14] M. Looke, M. F. Maloney, and S. P. Bell, "Mcm10 regulates DNA replication elongation by stimulating the CMG replicative helicase," Genes \& Development, vol. 31, no. 3, pp. 291305, 2017.

[15] Y. Takei and G. Tsujimoto, "Identification of a novel MCM3associated protein that facilitates MCM3 nuclear localization," Journal of Biological Chemistry, vol. 273, no. 35, pp. 2217722180, 1998.

[16] X. Liao, X. Liu, C. Yang et al., "Distinct diagnostic and prognostic values of minichromosome maintenance gene expression in patients with hepatocellular carcinoma," Journal of Cancer, vol. 9, no. 13, pp. 2357-2373, 2018.

[17] S. Li, Z. Jiang, Y. Li, and Y. Xu, "Prognostic significance of minichromosome maintenance mRNA expression in human lung adenocarcinoma," Oncology Reports, vol. 42, no. 6, pp. 2279-2292, 2019.

[18] H. Zhou, Y. Xiong, G. Zhang et al., "Elevated expression of minichromosome maintenance 3 indicates poor outcomes and promotes G1/S cell cycle progression,proliferation,migration and invasion in colorectal cancer," Bioscience Reports, vol. 40 , no. 7, 2020.

[19] H.-T. Li, B. Wei, Z.-Q. Li et al., "Diagnostic and prognostic value of MCM3 and its interacting proteins in hepatocellular carcinoma," Oncology Letters, vol. 20, no. 6, p. 1, 2020.

[20] S. Løkkegaard, D. Elias, C. L. Alves et al., "MCM3 upregulation confers endocrine resistance in breast cancer and is a predictive marker of diminished tamoxifen benefit," npj Breast Cancer, vol. 7, no. 1, p. 2, 2021.

[21] C. Kobierzycki, "Comparison of minichromosome maintenance proteins (MCM-3, MCM-7) and metallothioneins (MT-I/II, MT-III) expression in relation to clinicopathological data in ovarian cancer," Anticancer Research, vol. 33, no. 12, pp. 5375-5383, 2013.

[22] F. Rezazadeh, "Evaluation of the Ki-67 and MCM3 expression in cytologic smear of oral squamous cell carcinoma," Journal of Dentistry, vol. 18, no. 3, pp. 207-211, 2017.

[23] D. R. Rhodes, J. Yu, K. Shanker et al., “_ONCOMINE_ : a cancer microarray database and integrated data-mining platform," Neoplasia, vol. 6, no. 1, pp. 1-6, 2004. 
[24] Z. Tang, C. Li, B. Kang, G. Gao, C. Li, and Z. Zhang, "GEPIA: a web server for cancer and normal gene expression profiling and interactive analyses," Nucleic Acids Research, vol. 45, no. W1, pp. W98-w102, 2017.

[25] D. Pyeon, M. A. Newton, P. F. Lambert et al., "Fundamental differences in cell cycle deregulation in human papillomaviruspositive and human papillomavirus-negative head/neck and cervical cancers," Cancer Research, vol. 67, no. 10, pp. 46054619, 2007.

[26] T. Li, J. Fan, B. Wang et al., "TIMER: a web server for comprehensive analysis of tumor-infiltrating immune cells," Cancer Research, vol. 77, no. 21, pp. e108-e110, 2017.

[27] M. Uhlen, C. Zhang, S. Lee et al., "A pathology atlas of the human cancer transcriptome," Science, vol. 357, no. 6352, article eaan2507, 2017.

[28] Á. Nagy, A. Lánczky, O. Menyhárt, and B. Győrffy, “Author Correction: Validation of miRNA prognostic power in hepatocellular carcinoma using expression data of independent datasets," Scientific Reports, vol. 8, no. 1, 2018.

[29] D. S. Chandrashekar, B. Bashel, S. A. H. Balasubramanya et al., "UALCAN: a portal for facilitating tumor subgroup gene expression and survival analyses," Neoplasia (New York, N.Y.), vol. 19, no. 8, pp. 649-658, 2017.

[30] S. V. Vasaikar, P. Straub, J. Wang, and B. Zhang, "LinkedOmics: analyzing multi-omics data within and across 32 cancer types," Nucleic Acids Research, vol. 46, no. D1, pp. D956D963, 2018.

[31] P. Biewenga, M. R. Buist, P. D. Moerland et al., "Gene expression in early stage cervical cancer," Gynecologic Oncology, vol. 108, no. 3, pp. 520-526, 2008.

[32] L. Scotto, G. Narayan, S. V. Nandula et al., "Identification of copy number gain and overexpressed genes on chromosome arm 20q by an integrative genomic approach in cervical cancer: potential role in progression," Genes, Chromosomes and Cancer, vol. 47, no. 9, pp. 755-765, 2008.

[33] N. Gan, "Increase of Mcm3 and Mcm4 expression in cervical squamous cell carcinomas," European Journal of Gynaecological Oncology, vol. 31, no. 3, 2010.

[34] J. Sun, Z. Zhang, S. Bao et al., "Identification of tumor immune infiltration-associated lncRNAs for improving prognosis and immunotherapy response of patients with non-small cell lung cancer," Journal for Immunotherapy of Cancer, vol. 8, no. 1, p. e000110, 2020.

[35] A. Shiratori, K. Okumura, M. Nogami et al., "Assignment of the $49-\mathrm{kDa}$ (PRIM1) and 58-kDa (PRIM2A and PRIM2B) subunit genes of the human DNA primase to chromosome bands 1q44 and 6p11.1-p12," Genomics, vol. 28, no. 2, pp. 350-353, 1995.

[36] B. Yatsula, C. Galvao, M. McCrann, and A. S. Perkins, “Assessment of F-MuLV-induced tumorigenesis reveals new candidate tumor genes including Pecam1, St7, and Prim2," Leukemia, vol. 20, no. 1, pp. 162-165, 2006.

[37] D. Liu, X.-X. Zhang, B.-X. Xi et al., "Sine oculis homeobox homolog 1 promotes DNA replication and cell proliferation in cervical cancer," International Journal of Oncology, vol. 45, no. 3, pp. 1232-1240, 2014.

[38] C. Vigouroux, J.-M. Casse, S.-F. Battaglia-Hsu et al., "Methyl(R217)HuR and MCM6 are inversely correlated and are prognostic markers in non small cell lung carcinoma," Lung Cancer, vol. 89, no. 2, pp. 189-196, 2015. 